Acta Botanica Mexicana 67: 59-66 (2004)

\title{
NEOTIPIFICACIÓN DE BEHRIA TENUIFLORA GREENE (ALLIACEAE)
}

\author{
José Luis León de la Luz y José Juan Pérez Navarro \\ Herbario HCIB, Centro de Investigaciones Biológicas del Noroeste, S.C. \\ Apdo. postal 128, $23000 \mathrm{La} \mathrm{Paz,} \mathrm{Baja} \mathrm{California} \mathrm{Sur,} \mathrm{México}$
}

\section{RESUMEN}

Behria tenuiflora Greene es un taxon endémico del sector meridional de la península de Baja California, México. Las poblaciones naturales se distribuyen en un intervalo altitudinal que comprende desde la cima de las montañas a $2200 \mathrm{~m}$ de elevación hasta prácticamente el nivel del mar. En este trabajo se neotipifica a $B$. tenuiflora, ya que el material original se encuentra desaparecido. Se incluye una descripción detallada y una ilustración de la planta.

Palabras clave: Alliaceae, Baja California, Behria, Bessera, México, neotipificación.

\begin{abstract}
Behria tenuiflora Greene is an endemic taxon of the southern part of the Baja California peninsula, Mexico. The wild populations are distributed in an altitudinal range from the top of the mountains at $2200 \mathrm{~m}$ to practically the sea level. In this paper we neotypify $B$. tenuiflora since the type specimen is lost. A detailed description and an illustration of the plant are included.
\end{abstract}

Key words: Alliaceae, Baja California, Behria, Bessera, Mexico, neotipication.

\section{INTRODUCCIÓN}

El taxon Behria tenuiflora Greene (Alliaceae) fue descrito en 1886 como género monoespecífico, a partir de fragmentos de umbelas de plantas recolectadas en el extremo meridional de Baja California Sur ("San José del Cabo"). Se trata de una planta geófita que básicamente consiste de un cormo con túnica membranosa, a partir del cual nacen algunas hojas, y un escapo, en cuyo ápice se desarrolla una umbela con llamativas flores tubulares de color escarlata. Aunque en sus cápsulas se forman abundantes semillas, éstas presentan una baja tasa de germinación. De 
acuerdo con observaciones personales, su propagación y limitada dispersión parecen darse mayormente por gemación del cormo que por semillas.

Lenz (1971) discutió la historia nomenclatural que este taxon ha sufrido. Al principio, el mismo E. L. Greene consideró que podría tratarse de una planta afín al género Brodiaea Sm. Posteriormente, al contar con colectas de material completo, que permitió un estudio más profundo, y particularmente por las características del perianto, se le asignó al género Brevoortia Woot. Aún en la actualidad, autores como Ramírez Delgadillo (1992) la ubican en el género Bessera Schultes f., argumentando que tanto Behria como Bessera presentan las flores de color rojo brillante y los estambres exsertos con los filamentos unidos en la base formando una pequeña copa. Por su parte, Lenz (1971), basándose en las peculiaridades del perianto, en especial en su forma netamente tubular (y no parcialmente como en Bessera) y en el carácter exserto del estigma (cuyo tamaño en Bessera es menor que el de los estambres), la ratificó como perteneciente al género monoespecífico Behria. Con respecto a la familia de adscripción, Shreve y Wiggins (1964) y Wiggins (1980) la ubicaron en Amaryllidaceae y Espejo y López-Ferrari (1993) en Alliaceae, siguiendo el sistema propuesto por Dahlgren et al. (1985), criterio que se acepta en este trabajo.

Greene (1886) anotó en la descripción original de la especie que: “... the umbels are all we posses of this very beautiful and interesting new ally of Brodiaea. They are ticketed San Jose del Cabo, which means that they are from Cape San Lucas, or thereabouts, but the name of the collector is unknown. The fragment has been lying in the herbarium of the (California) Academy (of Science) for many years, and I have supposed before opening a perianth that the plant could be a second species of Brevoortia".

Lo anterior permite asumir que Greene revisó este material cuando estuvo asociado a la Academia de Ciencias de California en San Francisco, California, por lo que el holotipo debió quedar depositado allí mismo. La descripción de Behria tenuiflora (1886) apareció en la revista "Bulletin of the California Academy of Science", lo que apoya la noción de que el mencionado material original debió pertenecer al herbario de la Academia. En 1906, la ciudad de San Francisco fue víctima de un terremoto e incendio generalizado, mismo que afectó un importante volumen de ese herbario (Chickering, 1989). El actual herbario CAS incorporó la parte salvada, pero de acuerdo con su curador (Thomas F. Daniel, com. pers., 2002), su colección de tipos no posee dicho ejemplar. Aunque Moore (1953) señaló la presencia de una porción del holotipo en el herbario de la Universidad de California en Berkeley (UC), ésta tampoco se encuentra ahora en la colección respectiva (Barbara Ertter, com. pers., 2002). De no haberse depositado en la colección de San Francisco, aún cabe la posibilidad de haberlo hecho en el herbario de la Universidad de Notre Dame (NDG) en Indiana, Estados Unidos, sitio con el que 
Greene estuvo asociado permanentemente. Sin embargo, la actual curadora (Barbara J. Hellenthal, com. pers., 2001) desconoce la ubicación de éste y de muchos otros tipos de especies descritas por el mencionado botánico. En resumen, en la actualidad no existe el tipo nomenclatural de esta especie en ninguno de los herbarios en los que laboró su autor. Adicionalmente, de acuerdo con la cita original de Greene, un ejemplar fragmentario sirvió como referencia para la descripción, mismo que hoy día no se encuentra resguardado.

Tomando en cuenta las posibilidades de inexistencia o pérdida del holotipo, el código internacional vigente de nomenclatura botánica (Greuter et al., 2000) en los artículos 9.6 (p. 13) y 9.9 (p. 14) justifica la neotipificación del taxon.

A continuación se transcribe la descripción original de esta especie.

Behria tenuiflora Greene Bull. Calif. Acad. Sci. 2: 143. 1886.

Pedicells 8-15, very slender, an inch or two long; perianth 10 lines long, the supra-basal sac 3 lines broad, tubular portion hardly more than a line in diameter, the ovate-oblong teeth about a line long, erect or slightly spreading, brownish, apparently; whole body of the perianth bright scarlet; anthers linear-oblong, a line long, obtuse at each end, fixed by the middle, yellow; capsule ovate, a half inch long. Ticketed as "San Jose del Cabo", near of Cabo San Lucas, Lower California. Collector unknown.

De lo anterior, cabe señalar que el término "line" se refiere a la unidad 1/12 de pulgada $(=2 \mathrm{~mm})$. En esta descripción, además de referirse sólo a las estructuras florales, se incluyen algunas imprecisiones, tales como el color de los lóbulos que siempre son de color escarlata y no pardos, así como la omisión de la descripción de bandas amarillas en la parte basal y a lo largo de los segmentos del perianto.

Debido a lo escueto de la descripción original, enseguida se presenta una más detallada, basada esencialmente en el neotipo, mismo que procede de una localidad cercana a San José del Cabo, donde presumiblemente fue colectado el material referido por Greene en la descripción original.

\section{Behria tenuiflora Greene}

NEOTIPO: México, Baja California Sur, Mpio. Los Cabos, San José del Cabo, $5 \mathrm{~km}$ al este de San Bernabé por el camino rumbo a Palo Escopeta, 230' $\mathrm{N}$, $109^{\circ} 39^{\prime}$ W, 130 m de elevación. Noviembre 11 del 2000. Terrenos de lomeríos con suelo de tipo regosol, con textura arenoso-limosa, derivado de depósitos aluviales a partir de rocas graníticas. José Luis León de la Luz 9857 (neotipo (aquí designado) HCIB 15992; isoneotipos MEXU, SD, UAMIZ). 
Hierba perenne, geófita, de hasta $60 \mathrm{~cm}$ de alto (escapo); el tallo es un cormo subgloboso cubierto por catáfilos membranosos de color pardo-marrón, de su base aplanada surgen raíces adventicias cuando se presenta suficiente humedad edáfica, el cormo alcanza 2(3) $\mathrm{cm}$ de diámetro ecuatorial y 3(4) $\mathrm{cm}$ en su eje polar; hojas fasciculadas, aciculares, fistulosas, glabras, generalmente 4 (a veces 3 ), de hasta $60 \mathrm{~cm}$ de largo y $3 \mathrm{~mm}$ de ancho, decumbentes, con estrías longitudinales, el margen de cada estría posee pequeñas denticulaciones $(<0.1 \mathrm{~mm})$ a todo lo largo; inflorescencia escaposa, solitaria, generalmente única, pedúnculo cilíndrico a oval en corte transversal, glabro, sin estrías, erecto o algo curvado, de hasta $60 \mathrm{~cm}$ de largo; umbela simple, con 5 a 12 flores divaricadas a reclinadas, brácteas florales escariosas, subuladas, de hasta $6 \mathrm{~mm}$ de largo; pedicelos de 2 a $2.5 \mathrm{~cm}$ de largo; flores de color escarlata, semi-erectas, tubulares, zigomorfas, de hasta $2.5 \mathrm{~cm}$ de largo, sacciformes en su base, el perianto con 6 lóbulos desiguales, los mayores hasta de $4 \mathrm{~mm}$, con una banda amarillenta que recorre toda la longitud de los segmentos del perianto desde su base hasta el ápice del lóbulo; estambres 6, insertos en la base del tubo formando una pequeña copa, a partir de allí libres, típicamente exsertos y excediendo el perianto hasta en $1 \mathrm{~cm}$, filamentos filiformes, blanco-rosados, dilatados en la inserción, las anteras uniloculares, introrsas, de hasta $3 \mathrm{~mm}$ de largo, de color verde oscuro; pistilo uno, súpero, exserto y excediendo el perianto hasta en 1-1.5 cm, provisto de ginóforo de 2-3 $\mathrm{mm}$, ovario ovoide, trilocular, de $4-5 \mathrm{~mm}$ de largo por 2 mm de diámetro, óvulos de inserción parietal, 20 a 30, estilo filiforme, verde cuando fresco, de hasta $2 \mathrm{~cm}$ de largo, estigma apical, en forma de disco papiloso, de $1 \mathrm{~mm}$ de diámetro; el fruto es una cápsula loculicida de $13 \mathrm{~mm}$ de largo y $6 \mathrm{~mm}$ de diámetro, semillas más de 20, oscuras y lustrosas, irregularmente lenticulares, de hasta $1 \mathrm{~mm}$ de diámetro, aparentemente viables (Fig. 1).

Nombre común: se le conoce popularmente como cebollín de monte.

Ejemplares adicionales examinados: México. Baja California Sur, Mpio. La Paz, camino La Burrera-La Laguna, cañón de la Burrera, $22^{\circ} 33^{\prime} \mathrm{N}, 110^{\circ} 02^{\prime} \mathrm{W}$, 1200 m de elevación, 2.11.1985, José Luis León de la Luz 925 (HCIB 266); Mpio. Los Cabos, ca. Rancho Matancitas, $23^{\circ} 12^{\prime} \mathrm{N}, 110^{\circ} 07^{\prime} \mathrm{W}, 400 \mathrm{~m}$ de elevación, 30.10.1987, José Luis León de la Luz 3033 (HCIB 278); Mpio. La Paz, cerro al sur de la Playa San Pedrito, Todos Santos, $23^{\circ} 23^{\prime}$ N, $110^{\circ} 13^{\prime}$ W, 320 m de elevación, 10.09.1996, José Luis León de la Luz 7835 (HCIB 7538); Mpio. La Paz, cerro Pedregoso, Pichilingue, Sierra Riolítica, $24^{\circ} 16^{\prime} \mathrm{N}, 110^{\circ} 19^{\prime} \mathrm{W}, 30$ m de elevación, 7.10.1980, José Luis León de la Luz 9380 (HCIB 1275); Mpio. La Paz, camino a Puerto Cerralvo, $24^{\circ} 15^{\prime} \mathrm{N}, 110^{\circ} 17^{\prime} \mathrm{W}, 40$ m de elevación, 12.10.2000, José Juan Pérez Navarro 1360 (HCIB 15993); Mpio. La Paz, valle de La Laguna, Sierra de La Laguna, 233' N, 10959' W, 1730 m de elevación, 27.10.2000, José Luis León 


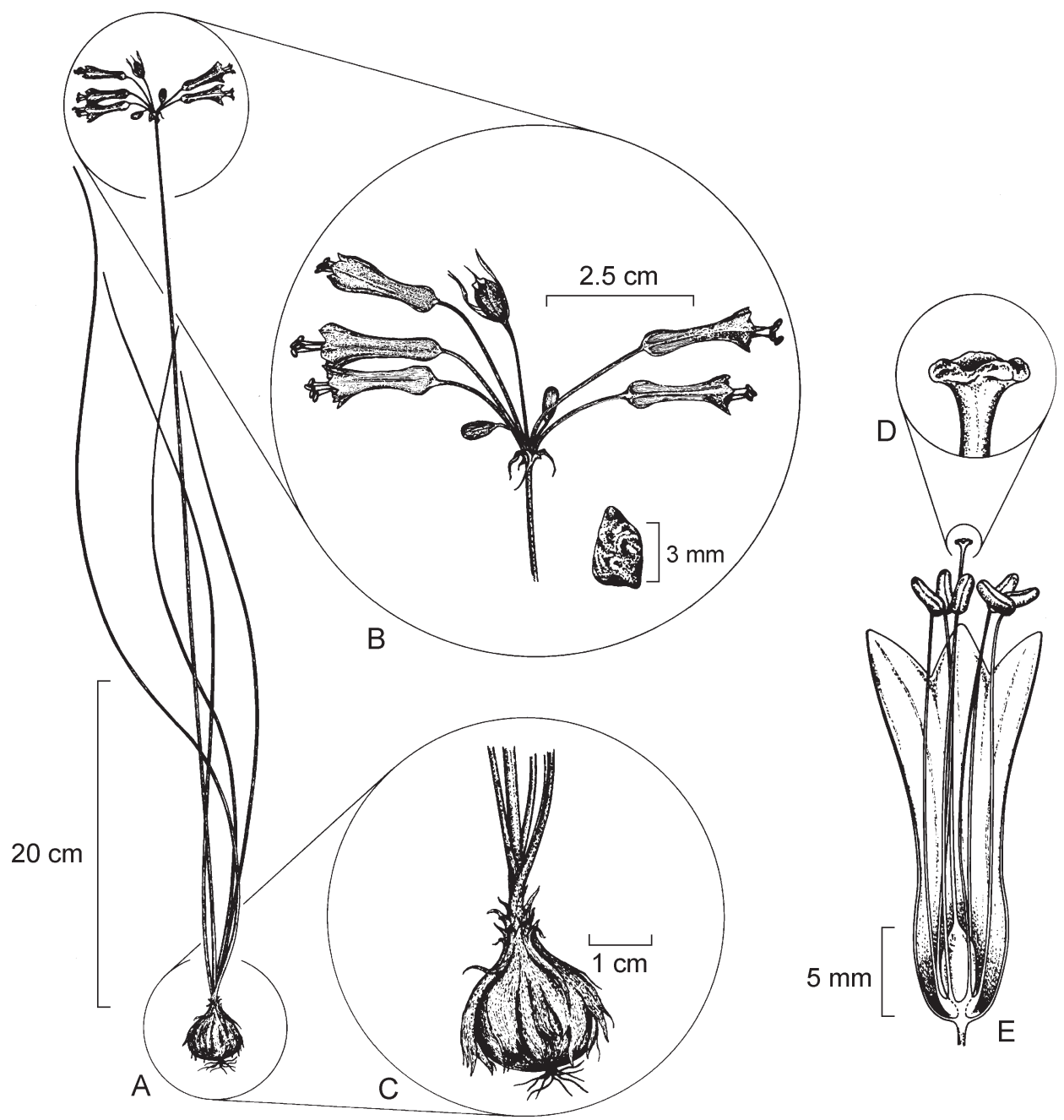

Fig. 1. Behria tenuiflora Greene. A. planta completa, con el cormo, cuatro hojas y el escapo con inflorescencias; B. acercamiento de la umbela, se observan las brácteas florales, flores exsertas y una cápsula, así como un acercamiento de una semilla; C. detalle de la túnica membranosa del cormo; D. detalle del estigma discoide papiloso; E. acercamiento de la flor, desprovista de una parte de la corola, en la cual se observa el ginóforo y la pequeña copa en la base de los estambres. Ilustración de Oscar Armendáriz Ruiz. 
de la Luz 9703 (HCIB 15188); Mpio. La Paz, Isla Partida, Isla Espíritu Santo, 243' N, 110²4' W; 60 m de elevación, 25.10.1996, José Luis León de la Luz 7964 (HCIB 7961); Mpio. La Paz, camino al cerro El Mechudo, estribaciones de la Sierra La Giganta, $24^{\circ} 47^{\prime}$ N, $110^{\circ} 40^{\prime}$ W, 30 m de elevación, 18.10.1996, Miguel Domínguez León 1388 (HCIB 7800).

La Fig. 2 muestra la distribución conocida de la especie. Las poblaciones de $B$. tenuiflora se establecen en forma dispersa sobre la superficie del extremo meridional de la península bajacaliforniana, principalmente dentro de lo que autores como León de la Luz et al. (1999) reconocen como la región del Cabo Peninsular. Es notable que esta especie se encuentra habitando en todas las comunidades vegetales reconocidas en esta porción de la península, que de acuerdo con León de la Luz et al. (1999) son bosque de pino-encino, bosque de encinos, selva baja caducifolia y matorral xerófilo en sus distintas variantes. Si bien la distribución principal de la especie corresponde al sustrato granítico propio de la región Capense, se han observado poblaciones aisladas, relativamente grandes, en las proximidades del Cerro Mechudo, en las islas San José y Espíritu Santo y en el área de Pichilingue

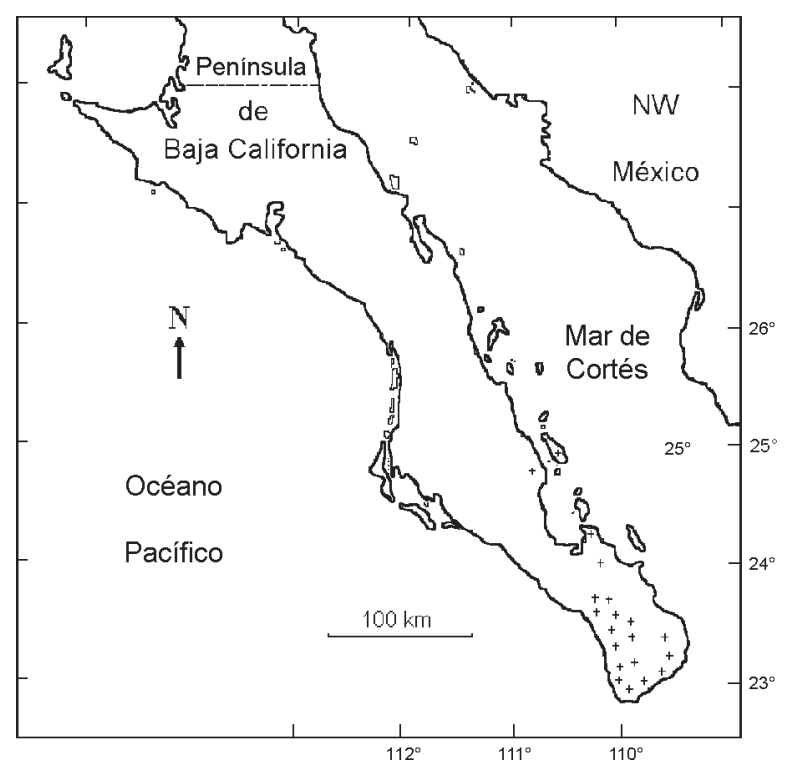

Fig. 2. Distribución geográfica conocida de Behria tenuiflora Greene en Baja California Sur, misma que coincide aproximadamente con la región del Cabo, desde el istmo de La Paz hacia el sur. 
al noreste de la ciudad de La Paz, todas estas localidades reconocidas como propias de la subdivisión del Desierto Sonorense, denominada Costa Central del Golfo.

En general los individuos de Behria conforman colonias compuestas por decenas de plantas que ocupan apenas unos cuantos metros cuadrados, pero en ocasiones las colonias pueden abarcar extensiones de una hectárea o más, con densidades de hasta cincuenta individuos por metro cuadrado. También se les puede encontrar como individuos solitarios, aislados por centenares de metros entre sí.

Moore (1953) menciona que existe polimorfismo entre las distintas poblaciones y es notorio que las características observadas en la población localizada en el bosque de pino-encino del Cerro San Antonio en la Sierra de la Laguna y las que muestra la población del matorral xerófilo de Pichilingue, representan los extremos de dicho polimorfismo, lo que sugiere la necesidad de efectuar un análisis taxonómico de esta especie. Además, la distribución de las poblaciones en superficies montañosas es más regular que en las zonas desérticas.

\section{AGRADECIMIENTOS}

Los autores agradecemos intensamente la participación del Sr. Miguel Domínguez León y del M. en C. Raymundo Domínguez Cadena su ayuda en el trabajo de campo y herbario. Al Dr. Adolfo Espejo Serna y M. en C. Ana Rosa López-Ferrari damos las gracias por su desinteresada colaboración al revisar críticamente la versión gruesa de este manuscrito.

\section{LITERATURA CITADA}

Espejo, A. y A. R. López-Ferrari. 1993. Las monocotiledóneas mexicanas, una sinopsis florística. Lista de referencia parte I (Agavaceae, Alismataceae, Alliaceae, Alstroemeriaceae y Amaryllidaceae). Consejo Nacional de la Flora de México, A.C. y Universidad Autónoma Metropolitana Iztapalapa, México D.F. 76 pp.

Chickering, S. 1989. Growing herbaria at the California Academy of Sciences. Fremontia 17(1): 3-19.

Dahlgren, R. M. T., H. T. Clifford y P. F. Yeo. 1985. The families of monocotyledons. Springer Verlag. Berlín. 520 pp.

Greene, E. L. 1886. Genera confused under Brodiaea. Bull. Calif. Acad. Sci. 2: 125-144.

Greuter, W. et al., 2000. International code of botanical nomenclature. Koeltz Scientific Book. Königstein. 474 pp.

Lenz, L. W. 1971. Two new species of Dandya (Liliaceae) from Mexico and a reexamination of Bessera and Behria. Aliso 7(3): 313-320. 
León de la Luz, J. L., J. J. Pérez Navarro, M. Domínguez y R. Domínguez. 1999. Flora de la Región del Cabo, Baja California Sur. Serie: Listados Florísticos de México. Vol. 18. Instituto de Biología, Universidad Nacional Autónoma de México. México, D.F. 39 pp.

Moore, H. E. 1953. The genus Milla (Amaryllidaceae-Alliaceae) and its allies. Gentes Herb. 8: 262-294.

Ramírez Delgadillo, R. 1992. Una nueva especie de Bessera (Liliaceae) del occidente de Jalisco, México. Boletín IBUG 3:131-136.

Shreve, F. e I. L. Wiggins. 1964. Vegetation and flora of the Sonoran Desert. Vol. 1. Stanford University Press. Stanford, California. 840 pp.

Wiggins, I. L. 1980. Flora of Baja California. Stanford University Press. Stanford, California. $1025 \mathrm{pp}$.

Recibido en agosto de 2001.

Aceptado en mayo de 2004. 